\title{
An economic lot and delivery scheduling problem with the fuzzy shelf life in a flexible job shop with unrelated parallel machines
}

\author{
S. Dousthaghi ${ }^{\mathbf{a}^{*}}$ and R. Tavakkoli-Moghaddam ${ }^{\mathrm{b}}$
}

${ }^{a}$ Department of Industrial Engineering, University of Economic Science, Tehran, Iran

${ }^{b}$ Department of Industrial Engineering, College of Engineering, University of Tehran, Tehran, Iran

\begin{tabular}{|c|c|}
\hline AR T I C L E I N F O & ABSTRACT \\
\hline $\begin{array}{l}\text { Article history: } \\
\text { Received } 25 \text { March } 2012 \\
\text { Accepted April } 32012 \\
\text { Available online } \\
5 \text { April } 2012 \\
\text { Keywords: } \\
\text { Lot and delivery scheduling } \\
\text { Flexible job shop } \\
\text { Unrelated parallel machines } \\
\text { Fuzzy shelf life }\end{array}$ & $\begin{array}{l}\text { This paper considers an economic lot and delivery scheduling problem (ELDSP) in a fuzzy } \\
\text { environment with the fuzzy shelf life for each product. This problem is formulated in a flexible } \\
\text { job shop with unrelated parallel machines, when the planning horizon is finite and it determines } \\
\text { lot sizing, scheduling and sequencing, simultaneously. The proposed model of this paper is based } \\
\text { on the basic period (BP) approach. In this paper, a mixed-integer nonlinear programming } \\
\text { (MINLP) model is presented and then it is changed into two models in the fuzzy shelf life. The } \\
\text { main model is dependent to the multiple basic periods and it is difficult to solve the resulted } \\
\text { proposed model for large-scale problems in reasonable amount of time; thus, an efficient heuristic } \\
\text { method is proposed to solve the problem. The performance of the proposed model is } \\
\text { demonstrated using some numerical examples. }\end{array}$ \\
\hline
\end{tabular}

\section{Introduction}

The purpose of a conventional economic lot and scheduling problem (ELSP) is to determine the cyclic production scheduling of different products on a single machine in lots, which minimizes the inventory holding and setup costs while demand is fulfilled without backlogging. This is a classical model of the ELSP originally presented by Roger (1958), where there are a number of contributions on the ELSP in the literature (Mokhlesian et al., 2010; Lütke entrupa et al., 2005; Liu et al., 2008). The ELSP with the delivery cost (ELDSP) is an extension of the conventional ELSP. To explain this problem, we may consider a supplier, which produces multiple components on a flexible job shop with unrelated parallel machines and then delivers them to an assembly facility. This model was presented by Hahm and Yano (1992) for the first time and after that they proposed two heuristics to solve the ELDSP under common cycle approach.

\footnotetext{
* Corresponding author. Tel: +989121580480

E-mail: sadegh.dousthaghi@ses.ac.ir (Sadegh Dousthaghi)

(C) 2012 Growing Science Ltd. All rights reserved. doi: $10.5267 /$ j.ijiec.2012.04.001
} 
To model the ELDSP, there are three well-known scheduling policies, namely common cycle approach (Hanssmann, 1962), basic period approach (Bomberger, 1966) and time varying lot size approach (Haessler, 1979). In this paper, the basic period approach is used for modeling the given problem.

In some real cases, manufacturers cannot store their products more than the product's shelf life time and if they need any product storage more than this shelf life time, some products such as food or beverages will be spoiled (Lütke entrupa et al., 2005). For this reason, some researchers have assumed this constraint in their ELSP's models (Silver, 1989; Sarker \& Babu, 1993; Sarker, 1994; Sliver, 1995; Viswanathan, 1995; Viswanathan \& Goyal, 1997; Soman et al., 2004; Lütke entrupa et al., 2005; Liu et al., 2008). Soman et al. (2004) proposed a model for finding better solution than the common cycle approach used for the ELSP problem. The method was based on a basic period approach and devised a heuristic to find promising solutions.

Lütke (2005) studied the single stage ELSP under the common cycle approach in yoghurt manufacturing by considering shelf life. Liu et al. (2008) presented a model for the ELSP under time varying lot size approach on a single stage production system. They assumed that all products have a shelf life and defined two separated models to solve the problem. In the first model, the zero-switchrule is considered and the second one is modeled without considering this rule. For solving these problems, a heuristic method has been proposed.

In recent years, researchers have had a great attention to the multi-stage production systems, such as flow shop and job shop (Tavakkoli-Moghaddam et al., 2011; Ziaeifar et al., 2011) and many researchers considered the ELSP and ELDSP in these production systems (El-Najdawi, 1993; Ouenniche \& Boctor, 1998; Ouenniche et al., 1999; Ouenniche \& Bertrand, 2001; Ouenniche \& Boctor, 2001a; Ouenniche \& Boctor, 2001b; Ouenniche \& Boctor, 2001c; Fatemi Ghomi \& Torabi, 2002; Torabi et al., 2005; Torabi et al., 2006; Jenabi et al., 2007; Torabi \& Jenabi, 2009a; Torabi \& Jenabi, 2009b).

In many real-world cases, products are scheduled for production in different stages. A flexible job shop (FJS) with unrelated parallel machines consists of two classical systems, namely job shop and parallel shop. This production system involves several work centers (i.e., production stages), in which one stage has at least one or more parallel unrelated machines. It is possible that this production system has same parallel machines in different stages; however, there is at least one unrelated parallel machine in at least one stage. Each product requires a sequence of operations in different stages based on its unique process route, and should be processed by at most one machine at each stage. However, some products may skip some stages. Jenabi et al. (2007) studied the ELDSP in the flexible flow shop with unrelated parallel machines based on common cycle approach and they presented a mixed-integer nonlinear programming (MINLP) model for this problem.

There are some studies about the ELSP and ELDSP on the job shop production environment. Ouenniche and Boctor (1998) studied the ELSP problem on the job shop production system and they presented a MINLP model to solve this problem under the common cycle approach. For solving the problem, they proposed two meta-heuristics, namely tabu search (TS) and simulated annealing (SA). Ouenniche and Bertrand (2001) worked on the ELSP on a job shop production environment based on the basic period approach. They proposed a new model for this problem and solved it by a heuristic method, namely multiple cycles (MC). In this method, they prepared an algorithm to produce all feasible basic period values, after that the sequencing vector and scheduling problem are considered by separate algorithms. TS and SA are used for sequencing the problem. Torabi et al. (2005) prepared an MINLP model for the ELSP under a common cycle approach in a flexible job shop with identical parallel machines, and they developed their model with sub-lots and zero setup costs separately. Finally, to solve the problem, they proposed an optimal solution. 
A fuzzy set theory is developed for solving the phenomenon of real-world cases that the fuzziness is prevalent for them. Up to now, there are so many studies about fuzzy problems and fuzzy sets (Pappis \& Karacapilidis, 1995; Vujosevic et al., 1996; Roy \& Maiti, 1997; Lee \& Yao, 1998; Lin \& Yao, 2000). In addition, many researchers have used the fuzzy sets and related assumptions to model their problem in the ELSP and ELDSP (Yao et al., 2005; Chang et al., 2006; Rao et al., 2009). Yao et al. (2005) investigated a model based on the common cycle approach for the ELSP with a fuzzy demand. They assumed that the demand for each product has a triangular membership function. They solved the problem with two basic methods, namely independent solution (IS) and common cycle (CC). Chang et al. (2006) studied the problem in a single machine based on the extended basic period and power-oftwo approach. They assumed that the demand for each product follows a triangular membership function. They proposed a genetic algorithm (GA) to solve the problem and compared the results of the algorithm with some lower bounds. Rao et al. (2009) investigated the ELSP in a single machine environment and multi products based on the basic period approach. They assumed the problem in a fuzzy environment with fuzzy inventory holding costs where it results to the fuzzy objective goal and fuzzy constraints. They proposed a fuzzy genetic approach to solve the mentioned problem.

To determine the parameters and values (e.g., demand rate and inventory holding cost), there is always some errors or deviations. Thus, researchers are interested in assuming some uncertainty associated with some parameters. Because of this reason, in this paper, the shelf life time is assumed as a fuzzy number, which has a triangular fuzzy membership function. To the best our knowledge, there is no contribution for the economic lot and delivery scheduling problem in a flexible job shop with unrelated parallel machines and a fuzzy shelf life under the basic period approach. Thus, in this paper, a new MINLP model and efficient solution is developed for this fuzzy problem.

The remainder of this paper is as follows. Section 2 presents the main model of the problem, problem description and notations used for the problem formulation, and then the basic model is developed to the fuzzy models. Section 3 proposes the heuristic method for this problem. The lower bound computations, experimental results are illustrated in Sections 4. Finally, conclusions are discussed in Section 5 .

\section{Problem description}

In this section, a new mixed-integer nonlinear programming (MINLP) model is presented to find the lot sizing, scheduling and sequencing, simultaneously.

\subsection{Model assumptions}

The following assumptions are considered for the given problem.

- At different stages, machines are continuously available, in which each machine can have only one operation at a time.

- Setup times and costs are sequence independent.

- Inventory holding costs of the end products and semi-finished products have been considered linearly.

- Preemption, lot streaming and backlogging are not allowed.

- There are unlimited intermediate buffers between stages.

- Production rate of different stages is enough to meet the demands rate and have feasible solutions.

- Zero switch rule is used (i.e., the component production starts at each basic period when its inventory level reaches zero).

- Each product has a fuzzy shelf life. 


\subsection{Notations and parameters}

$n \quad$ Number of products

$m \quad$ Number of work centers or production stages

$i, u \quad$ Product indices

$j \quad$ Stage index

$m_{i} \quad$ Number of required operations to produce product $i$

$M_{j} \quad$ Number of parallel machines in stage $j$

$n_{j} \quad$ Number of products that should be produced in stage $j$

$M_{k^{\prime} j} \quad k^{\prime}$-th machine in stage $j$

$\mu(i, 0) \quad$ Production route of product $i$

$P(j) \quad$ Set of products processed at stage $j$

$\mu(i, r) \quad r$-th stage on the route of product $i$

$d_{i} \quad$ Demand rate of product $i$

$p_{i, k^{\prime}, \mu(i, r)}$ Production rate of product $i$ on machine $k^{\prime}$ at the $r$-th stage on its route

$S t_{i, k^{\prime}, \mu(i, r)}$ Sequence independent setup time of product $i$ on machine $k^{\prime}$ at the $r$-th stage on its route

$S_{i, k^{\prime}, \mu(i, r)}$ Sequence independent setup cost of product $i$ on machine $k^{\prime}$ at the $r$-th stage on its route

$A \quad$ Transportation cost per delivery

$h_{i, \mu(i, r)} \quad$ Inventory holding cost per a unit of product iper time unit between the $r$-th and $(r+1)$-th stages on its route

$h_{i} \quad$ Inventory holding cost per a unit of final product $i$ per unit time

$\mathrm{PH} \quad$ Planning horizon length

$L_{i} \quad$ Shelf life of product $i$ in time unit

$M \quad$ A large real number

\subsection{Decision variables}

$\sigma_{k} \quad$ Production sequence vector of the $k$-th basic period

$\sigma_{k k^{\prime} j} \quad$ Production sequence vector of machine $k^{\prime}$ at stage $j$ in the $k$-th basic period

$R \quad$ Number of production cycles over the finite planning horizon

$F \quad$ The basic period length

$k_{i} \quad$ Time multiple of product $i$

$J_{k^{\prime} j} \quad$ Set of products which are assigned to machine $M_{k^{\prime} j}$

$n_{k k^{\prime} j} \quad$ Number of products which are assigned to machine $M_{k^{\prime} j}$ in the $k$-th basic period

$b_{i, \mu(i, r)} \quad$ Process beginning time of product $i$ at the $r$-th stage on its route (after setup operations)

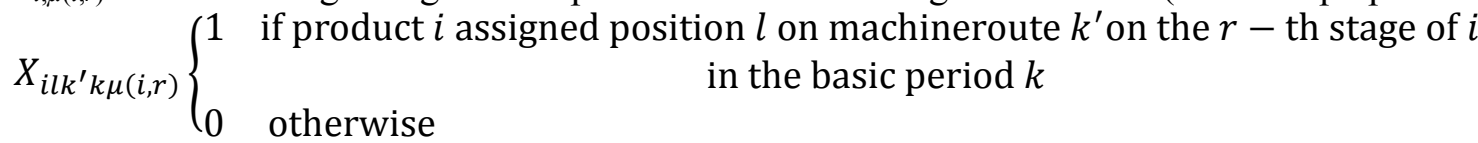

$H \quad$ Least common multiple (LCM) of $k_{i}$ values $\left(k_{1}, k_{2}, \ldots, k_{n}\right)$

$T_{i} \quad$ Production cycle length $\left(T_{i}=k_{i} \times F\right)$

\subsection{Basic model}

For modeling the objective function of the ELDSP, three main terms are assumed. The first and the second terms are the setup cost and delivery cost of lot sizes. The third term computes the inventory holding cost of products. The delivery costs and setup costs per a unit time are evaluated as follows:

$C_{1}=\frac{\sum_{i=1}^{n} \sum_{r=1}^{m_{i}} \sum_{k^{\prime}=1}^{m_{j}} s_{i k^{\prime} \mu(i, r)} \cdot \sum_{l=1}^{n_{j}} X_{i l k^{\prime} k \mu(i, r)}}{k_{i} \cdot F}+\frac{A}{F}$ 
The inventory holding cost has three subsets: The first one calculates the holding cost of work-inprocess (WIP) inventory. The second and third ones calculate the inventory holding costs of the supplier's final products and assembler's final products, respectively. Fig. 1 illustrates the inventory level of WIP inventory.

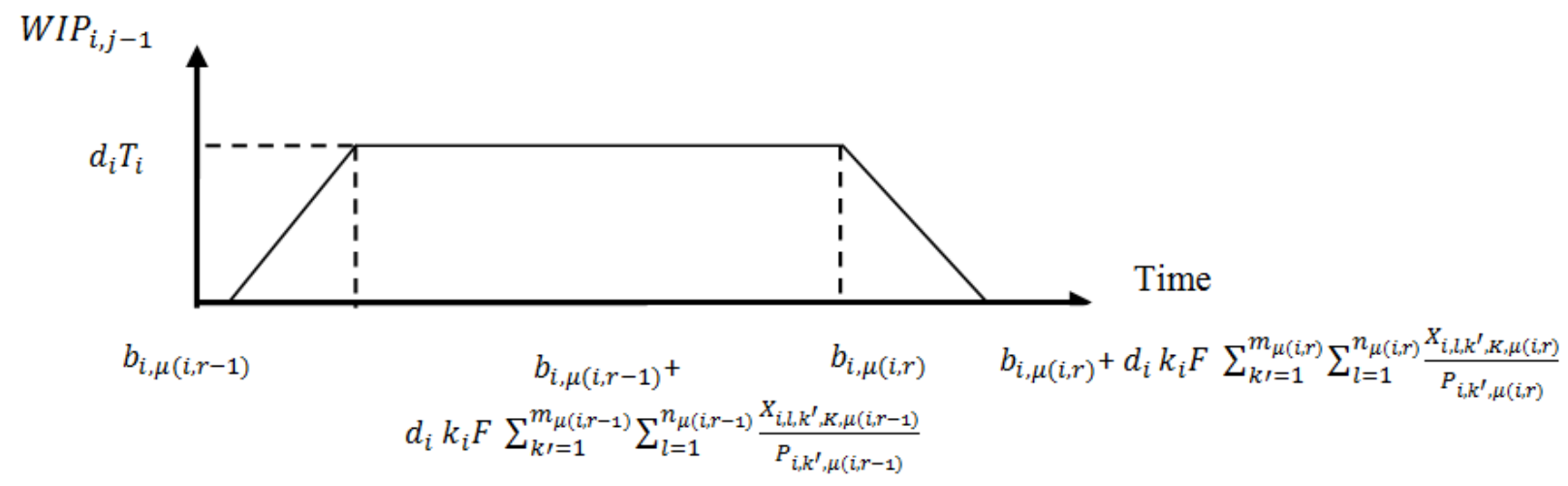

Fig. 1. WIP between stages $\mu_{(i, r-1)}$ and $\mu_{(i, r)}$

Thus, the average of the WIP inventory level between stages is as follows,

$$
\begin{gathered}
I_{i, j-1}=\frac{d_{i} T_{i}}{T_{i}}\left(b_{i, \mu(i, r)}+d_{i} T_{i} \sum_{k^{\prime}=1}^{m_{\mu(i, r)}} \sum_{l=1}^{n_{\mu(i, r-1)}} \frac{X_{i, l, k^{\prime}, k, \mu(i, r)}}{2 P_{i, k^{\prime}, \mu(i, r)}}-b_{i, \mu(i, r-1)}\right. \\
\left.-d_{i} T_{i} \sum_{k^{\prime}=1}^{m_{\mu(i, r)}} \sum_{l=1}^{n_{\mu(i, r)}} \frac{X_{i, l, k^{\prime}, k, \mu(i, r-1)}}{2 . P_{i, k^{\prime}, \mu(i, r-1)}}\right)
\end{gathered}
$$

Therefore, the WIP inventory holding cost of products per a unit time is computed by:

$$
\begin{gathered}
T C_{W I P}=\sum_{i=1}^{n} \sum_{r=2}^{m_{i}} h d_{i, \mu(i, r-1)}\left(b_{i, \mu(i, r)}+d_{i} T_{i} \sum_{k^{\prime}=1}^{m_{\mu(i, r)}} \sum_{l=1}^{m_{\mu(i, r-1)}} \frac{X_{i, l, k^{\prime}, k, \mu(i, r)}}{2 P_{i, k^{\prime}, \mu(i, r)}}-b_{i, \mu(i, r-1)}\right. \\
\left.\quad-d_{i} T_{i} \sum_{k^{\prime}=1}^{n_{\mu(i, r)}} \sum_{l=1}^{X_{i, l, k^{\prime}, k, \mu(i, r-1)}} \frac{P_{i, k^{\prime}, \mu(i, r-1)}}{2 .}\right)
\end{gathered}
$$

In Fig. 2, the inventory level of supplier's final products is illustrated.

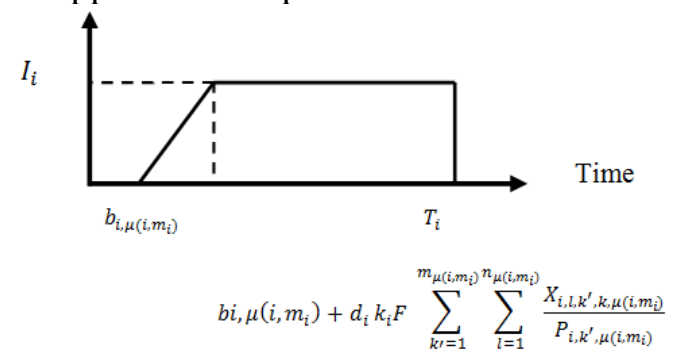

Fig. 2. Final product inventory level at supplier's storage

Therefore, when WIP inventory holding cost is computed, the average inventory holding cost of all products in supplier's storage is as follows: 
$T C_{F I}=\sum_{i=1}^{n} h_{i} d_{i}\left(1-\frac{1}{2} \sum_{k^{\prime}=1}^{m_{\mu\left(i, m_{i}\right)}} \sum_{l=1}^{n_{\mu\left(i, m_{i}\right)}} \frac{X_{i, l, k^{\prime}, k, \mu\left(i, m_{i}\right)}}{P_{i, k^{\prime}, \mu\left(i, m_{i}\right)}}\right) k_{i} F-\sum_{i=1}^{n} h_{i} d_{i} b_{i, \mu\left(i, m_{i}\right)}$

Fig. 3 shows the inventory level of the final product in one cycle at the assembly facility. The average inventory holding cost for all products per unit time at the assembler storage is computed by:

$$
T C_{A S M}=\frac{\boldsymbol{k}_{\boldsymbol{i}} \boldsymbol{F}}{\mathbf{2}} \sum_{\boldsymbol{i}=\mathbf{1}}^{\boldsymbol{n}} \boldsymbol{h}_{\boldsymbol{i}} \boldsymbol{d}_{\boldsymbol{i}}
$$

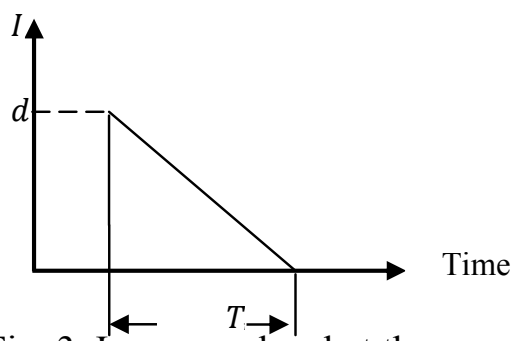

Fig. 3. Inventory level at the assembler in one cycle

Consequently, the objective function of the problem is equal to the summation of three main terms explained earlier. Therefore, the objective function is equal to Eq. (6) with some considerations. The constraints of the basic ELDSP model in a flexible job shop with unrelated parallel machines without considering the fuzzy shelf life are shown below.

\section{Problem $\boldsymbol{P}$}

Min

$Z=\frac{\sum_{i=1}^{n} \sum_{r=1}^{m_{i}} \sum_{k^{\prime}=1}^{m_{j}} S_{i k^{\prime} \mu(i, r)} \cdot \sum_{l=1}^{n_{j}} X_{i, l, k^{\prime}, K, \mu\left(i, m_{i}\right)}}{k_{i} \cdot F}+\frac{A}{F}+$

$\sum_{i=1}^{n} \frac{d_{i}{ }^{2}}{2}\left(k_{i} F\right) \cdot \sum_{r=2}^{m_{i}} h_{i, \mu(i, r-1)}\left(\sum_{k^{\prime}=1}^{m_{j}} \sum_{l=1}^{n_{j}} \frac{X_{i, l, k^{\prime}, k, \mu(i, r)}}{p_{i, k^{\prime}, \mu(i, r)}}-\right.$

$\left.\sum_{k^{\prime}=1}^{m_{j-1}} \sum_{l=1}^{n_{j}} \frac{X_{i, l, k^{\prime}, k, \mu(i, r-1)}}{p_{i, k^{\prime}, \mu(i, r-1)}}\right)+\sum_{i=1}^{n} \sum_{r=2}^{m_{i}} h_{i, \mu(i, r-1)} \cdot d_{i}\left(b_{i, \mu(i, r)}-b_{i, \mu(i, r-1)}\right)+F \cdot \sum_{i=1}^{n} \frac{h_{i} d_{i} k_{i}}{2}(3-$

$\frac{\sum_{k^{\prime}=1}^{m_{m}} \sum_{l=1}^{n_{j}} X_{i, l, k^{\prime}, k, \mu(i, r-1)}}{p_{i, k^{\prime}, m_{i}}}-\sum_{i=1}^{n} h_{i} d_{i} b_{i, \mu\left(i, m_{i}\right)}$

subject to

$$
\begin{aligned}
& b_{i, \mu(i, r-1)}+d_{i} . k_{i} . F \cdot \sum_{k^{\prime}=1}^{m_{j}} \sum_{l=1}^{n_{j}} \frac{X_{i, l, k^{\prime}, k_{i}, \mu(i, r-1)}}{P_{i, k^{\prime}, \mu(i, r-1)}} \leq b_{i, \mu(i, r)} ; i=1, \ldots, n ; r=2, \ldots, m_{i} \\
& b_{i, \mu(i, r)}+\frac{d_{i}, k_{i} \cdot F}{P_{i, k^{\prime}, \mu(i, r)}}+S t_{u, k^{\prime}, \mu(i, r)}-b_{u, \mu(i, r)} \leq M\left(2-X_{i, l, k^{\prime}, k, \mu(i, r)}-X_{u,(l+1), k^{\prime}, k, \mu(i, r)}\right) ; i \neq u, i, u \\
& \in p(j) ; L<n_{j} ; r=1, \ldots, m_{i} ; k^{\prime}=1, \ldots, m_{j} ; k=1, \ldots, H ; H=L C M\left(k_{1}, k_{2}, \ldots, k_{n}\right) \\
& \sum_{i \in p(j)}^{n} X_{i, l, k^{\prime}, k, j} \leq 1 ; k^{\prime}=1, \ldots, m_{j} ; l=1, \ldots, n_{j} ; k=1, \ldots, H ; H=L C M\left(k_{1}, k_{2}, \ldots, k_{n}\right) ; j= \\
& 1, \ldots, m_{i} \\
& \sum_{i \in p(j)}^{n} X_{i, l+1, k^{\prime}, k, j} \leq \sum_{u \in p(j)}^{n} X_{u, l, k^{\prime}, k, j} ; k^{\prime}=1, \ldots, m_{j} ; l=1, \ldots, n_{j} ; k=1, \ldots, H ; H= \\
& L C M\left(k_{1}, k_{2}, \ldots, k_{n}\right) \\
& \sum_{k^{\prime}=1}^{m_{j}} \sum_{l=1}^{n_{j}} \sum_{k=1}^{k_{i}} X_{i, l, k^{\prime}, k, j}=1 ; i \in p(j) ; j=1, \ldots, m_{i}
\end{aligned}
$$




$$
\begin{aligned}
& \sum_{k^{\prime}=1}^{m_{j}} \sum_{l=1}^{n_{j}} X_{i, l, k^{\prime},\left(t+b k_{i}\right), j}=\sum_{k^{\prime}=1}^{m_{j}} \sum_{l=1}^{n_{j}} X_{i, l, k^{\prime},\left(t+(b+1) k_{i}\right), j} ; i \in p(j) ; j=1, \ldots, m_{i} ; t=1, \ldots, k_{i} ; b \\
& \quad=0, \ldots, \frac{H}{k_{i}}-2 \\
& \sum_{k^{\prime}=1}^{m_{j}} \sum_{l=1}^{n_{j}} X_{i, l, k^{\prime}, k, \mu(i, r)}=\sum_{k^{\prime}=1}^{m_{j}} \sum_{l=1}^{n_{j}} X_{i, l, k^{\prime}, k, \mu(i, r+1)} ; i \in p(j) ; r=1, \ldots, m_{i} ; r<m ; k=1, \ldots, H \\
& b_{i, \mu(i, r)} \geq \sum_{k^{\prime}=1}^{m_{\mu(i, r)}} S t_{i, k^{\prime}, \mu(i, r)} . X_{i, l, k^{\prime}, k, \mu(i, r)}-M\left(1-\sum_{k^{\prime}=1}^{m_{\mu(i, r)}} X_{i, l, k^{\prime}, k, \mu(i, r)}\right) ; r=1, \ldots, m_{i} ; i \in \\
& n ; k=1, \ldots, H ; \\
& H=L C M\left(k_{1}, k_{2}, \ldots, k_{n}\right) \\
& F \leq \frac{L_{i}}{k_{i} \cdot\left(1-\sum_{k^{\prime}=1}^{m_{j}} \sum_{r=1}^{m_{i}} \sum_{l=1}^{n_{j}} \frac{X_{i, l, k^{\prime}, k, \mu(i, r)} \times d_{i}}{p_{i, k^{\prime}, \mu(i, r)}}\right)} ; i=1, \ldots, n ; k=1, \ldots, H ; H=L C M\left(k_{1}, k_{2}, \ldots, k_{n}\right) \\
& b_{i, \mu\left(i, m_{i}\right)}+\sum_{k^{\prime}}^{m_{j}} \sum_{l=1}^{n_{j}} \frac{d_{i} k_{i} F \cdot X_{i, l, k^{\prime}, k, \mu\left(i, m_{i}\right)}}{p_{i, k \prime, \mu\left(i, m_{i}\right)}} \leq F ; i=1, \ldots, n \\
& H . F . R=P H ; H=L C M\left(k_{1}, k_{2}, \ldots, k_{n}\right) \\
& R \geq 1 ; R=\operatorname{integer} \\
& F \geq 0 ; b_{i, j} \geq 0 \forall i, j ; X_{i, l, k^{\prime}, k, j} \in\{1,0\} ; \forall i, j, k^{\prime}, k, l
\end{aligned}
$$

In this section, a brief explanation for this model is given. Constraint (7) shows that all products can be processed at the next stage on their routes if and only if their lot size operations are completed at the previous stage. Constraint (8) ensures that each product can be processed at each stage after the completion of its predecessor associated with production sequence. Constraint (9) reveals that there is at most one product at each stage, at each parallel machine, at each basic period and at each position of the sequence. Constraint (10) assures that no product can be assigned at the $(l+1)$ position of machine $M_{k^{\prime}}$ if and only if another product is assigned in the previous position $(l)$ of this product sequence.

Constraint (11) ensures that at each stage and for each product, solutions of the model have unique product assignment to the parallel machines in their sequencing positions. Constraint (12) states that the products are assigned to different basic periods during the $H$ basic period, perfectly. Constraint (13) shows that when a product is assigned to the basic period $k$ at stage $j$, this product should be assigned to all stages before and after the $j$-th stage on its route in this basic period. Constraint (14) ensures that if a product $i$ is assigned to one of the parallel machines on stage $j$, then the production start time of product $i$ should be greater than setup time of this product. Constraint (15) states that the total production time of product $i$ and the storage time of this product should be smaller than the shelf life time of product $i$. Constraint (16) ensures that the total production time and setup time of each product should be smaller than the basic cycle time $F$. Constraint (17) states that the planning horizon is an integer multiple of $H$ and $F$. Constraint (19) is the no-negativity of variables.

\subsection{Fuzzy model}

In this section, the basic model is modified with the fuzzy shelf life values. Since the fuzzy shelf life for product is given as an approximate $l_{i 0}$ with the triangular membership function, the equivalent of $\alpha$-cut is as follows: 


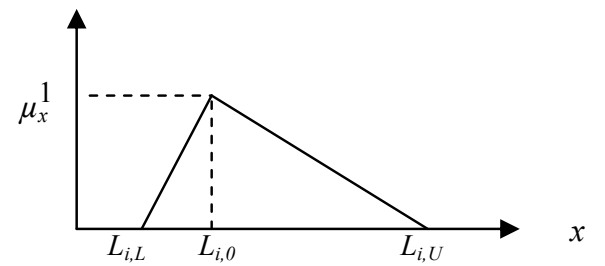

Fig. 4. Fuzzy triangular membership function

$$
u_{\widetilde{L_{l}}}=\left\{\begin{array}{l}
\frac{\left(L_{i}-L_{i, L}\right)}{\left(L_{i, 0}-L_{i, L}\right)} L_{i, L} \leq L_{i} \leq L_{i, 0} \\
\frac{\left(L_{i, U}-L_{i}\right)}{\left(L_{i, U}-L_{i, 0}\right)} L_{i, 0} \leq L_{i} \leq L_{i, U} \\
0 \quad \text { Otherwise }
\end{array} \rightarrow L_{i}^{\mathrm{a}}=\left[\left(L_{i, 0}-L_{i, L}\right) a+L_{i, L}, L_{i, U}-a\left(L_{i, U}-L_{i, 0}\right)\right]\right.
$$

With this equation, the shelf life constraint is changed into two constraints of the lower bound and upper bound of the shelf life as follows,

$$
\begin{aligned}
& F_{L}=\frac{\left(L_{i, 0}-L_{i, L}\right) \alpha+L_{i, L}}{\left.k_{i \cdot\left(1-\sum_{k^{\prime}=1}^{m_{j}} \sum_{j=1}^{m_{i}} \frac{X_{i, l, k^{\prime}, k, \mu(i, r)} \times d_{i}}{p_{i, k \prime}, \mu\left(i, m_{i}\right)}\right.}\right)}, \\
& \left.F_{U}=\frac{L_{i, U}-\alpha\left(L_{i, U}-L_{i, 0}\right)}{k_{i} \cdot\left(1-\sum_{k^{\prime}=1}^{m_{j}} \sum_{j=1}^{m_{i}} \frac{X_{i, l, k^{\prime}, k, \mu(i, r)} \times d_{i}}{p_{i, k \prime}, \mu\left(i, m_{i}\right)}\right.}\right)
\end{aligned}
$$

Therefore, with Eq. (21) and Eq. (22), the basic model is changed in the objective function and some constraints. Consequently, there are two fuzzy models for the lower and upper bounds called $P_{f l}$ and $P_{f u}$, respectively. Thus, there are some changes in the basic model in order to reach to the fuzzy models. In this section, these changes are illustrated for the upper bound model or $P_{f u}$.

\section{Model $P_{f u}$ :}

$\min Z_{\alpha}^{i U} \cong \mathrm{TC}=\frac{\sum_{i=1}^{n} \sum_{r=1}^{m_{i}} \sum_{k^{\prime}=1}^{m_{j}} S_{i, k^{\prime}, \mu(i, r)} \cdot \sum_{l=1}^{n_{j}} X_{i, l, k^{\prime}, k, \mu\left(i, m_{i}\right)}}{k_{i} \cdot F_{\alpha}^{U}}+$

$\frac{A}{F_{\alpha}^{U}}+\sum_{i=1}^{n} \frac{d_{i}^{2}}{2}\left(k_{i} F_{\alpha}^{U}\right) \cdot \sum_{r=2}^{m_{i}} h_{i, \mu(i, r-1)}\left(\sum_{k^{\prime}=1}^{m_{j}} \sum_{l=1}^{n_{j}} \frac{X_{i, l, k^{\prime}, k, \mu(i, r)}}{p_{i, k^{\prime}, \mu(i, r)}}-\sum_{k^{\prime}=1}^{m_{j-1}} \sum_{l=1}^{n_{j}} \frac{X_{i, l, k^{\prime}, k, \mu(i, r-1)}}{p_{i, k^{\prime}, \mu(i, r-1)}}\right)+$

$\sum_{i=1}^{n} \sum_{r=2}^{m_{i}} h_{i, \mu(i, r-1)} \cdot d_{i}\left(b_{i, j}-b_{i, j-1}\right)+F_{\alpha}^{U} \cdot \sum_{i=1}^{n} \frac{h_{i} d_{i} k_{i}}{2}\left(3-\frac{\sum_{k^{\prime}=1}^{m_{m}} \sum_{l=1}^{n_{j}} X_{i, l, k^{\prime}, k, \mu(i, r-1)}}{p_{i, k^{\prime}, m_{i}}}\right)-$

$\sum_{i=1}^{n} h_{i} d_{i} b_{i, \mu}\left(i, m_{i}\right)$

subject to

$$
\begin{aligned}
& b_{i, \mu(i, r-1)}+d_{i \cdot} k_{i} \cdot F_{\alpha}^{U} \cdot \sum_{k^{\prime}=1}^{m_{j}} \sum_{l=1}^{n_{j}} \frac{X_{i, l, k^{\prime}, k_{i}, \mu(i, r-1)}}{P_{i, k^{\prime}, \mu(i, r-1)}} \leq b_{i, \mu(i, r)} ; i=1, \ldots, n ; r=2, \ldots, m_{i} \\
& b_{i, \mu(i, r)}+\frac{d_{i \cdot k_{i} \cdot F_{\alpha}^{U}}}{P_{i, k^{\prime}, \mu(i, r)}}+S t_{u, k^{\prime}, \mu(i, r)}-b_{u, \mu(i, r)} \leq \mathrm{M}\left(2-X_{i, l, k^{\prime}, k, j}-X_{u,(l+1), k^{\prime}, k, j}\right) ; i \neq \mathrm{u}, i, \mathrm{u} \in \mathrm{p}(r) ; \mathrm{L} \\
& <n_{j} \\
& F_{\alpha}^{U} \leq \frac{L_{i, U}-\alpha\left(L_{i, U^{-}}-L_{i, 0}\right)}{\left.k_{i \cdot\left(1-\sum_{k^{\prime}=1}^{m_{j}} \sum_{j=1}^{m_{i}} X_{i, l, k^{\prime}, k, \mu(i, r)} \times d_{i}\right.}^{P_{i, k^{\prime}, \mu\left(i, m_{i}\right)}}\right)} i=1, \ldots, n ; j=2, \ldots, m_{i}
\end{aligned}
$$


$b_{i, \mu\left(i, m_{i}\right)}+\sum_{k^{\prime}}^{m_{j}} \sum_{l=1}^{n_{j}} \frac{d_{i} k_{i} F_{\alpha}^{U} \cdot X_{i, l, k^{\prime}, k, \mu\left(i, m_{i}\right)}}{p_{i, k l, \mu\left(i, m_{i}\right)}} \leq F_{\alpha}^{U} ; i=1, \ldots, n$

H. $F_{\alpha}^{U} . r=P H ; H=\operatorname{LCM}\left(k_{1}, k_{2}, \ldots, k_{n}\right)$

$R \geq 1 ; R=$ integer $; 0 \leq a \leq 1 F_{\alpha}^{U} \geq 0 ; b_{i, j} \geq 0 \forall i, j ; X_{i, l, k^{\prime}, k, j} \in\{1,0\} ; \forall i, j, k^{\prime}, k, I$

In the $P_{f u}$ model, the constraints are changed and illustrated. Other constraints are the same as Eq. (9) to Eq. (14). The computation of the lower bound fuzzy model or $P_{f l}$ is as the same as $P_{f u}$ with changing the parameters to lower bound parameters. These two proposed fuzzy models determine lot sizing, sequencing and scheduling of products, simultaneously. Because the proposed model depends on the $k_{i}$ values, for solving this MINLP model with the exact methods, we have to determine $k_{i}$ values first. Therefore, in the next section, a heuristic algorithm is described to solve the problems $P_{f u}$ and $P_{f l}$.

\section{Solution procedure}

The MINLP model presented in this paper can be solved with different heuristics or meta-heuristics. In this paper, a heuristic method is defined, whose details are presented in the next sections.

\subsection{Determining a list of candidate vectors of $k_{i}$ values}

In this step, an algorithm is explained, which results to the all of the candidate vectors of the multipliers. The algorithm is defined as follows.

Step 1: This step needs to determine the $T^{i *}$ as an optimal independent cycle time. The objective function is as follows,

$T C^{i *}=\frac{\sum_{r=1}^{m_{i}} S_{i k^{\prime} \mu(i, r)}}{T_{i}}$

$\frac{d_{i}{ }^{2}}{2}\left(T_{i}\right) \cdot \sum_{r=2}^{m_{i}} h_{i, \mu(i, r-1)}\left(\frac{1}{p_{i, k^{\prime}, \mu(i, r)}}-\frac{1}{p_{i, k^{\prime}, \mu(i, r-1)}}\right)+\sum_{r=2}^{m_{i}} h_{i, \mu(i, r-1)} \cdot d_{i}\left(b_{i, \mu(i, r)}-b_{i, \mu(i, r-1)}\right)$

$T_{i} \frac{h_{i} d_{i}}{2}\left(3-\frac{1}{p_{i k^{\prime} \mu\left(i, m_{i}\right)}}\right)-h_{i} d_{i} b_{i, \mu\left(i, m_{i}\right)}$

To determine this value from Eq. (7) and Eq. (16), the following changes are performed to Eq. (30),

$$
\begin{aligned}
& T C^{i *}=\frac{\sum_{r=1}^{m_{i}} \sum_{k^{\prime}=1}^{m_{j}} s_{i k^{\prime} \mu(i, r)}}{T_{i}}+T_{i} \times \\
& \left(\frac{d_{i}^{2}}{2} \sum_{r=2}^{m_{i}} h_{i, \mu(i, r-1)}\left(\sum_{k^{\prime}=1}^{m_{j}} \frac{1}{p_{i, k^{\prime}, \mu(i, r)}}+\sum_{k^{\prime}=1}^{m_{j-1}} \frac{1}{p_{i, k^{\prime}, \mu(i, r-1)}}\right)+\frac{h_{i} d_{i}}{2}\left(3-\frac{1}{p_{i k^{\prime} \mu\left(i, m_{i}\right)}}\right)-\right. \\
& \left.h_{i} d_{i}^{2} \sum_{r=1}^{m_{i-1}} \sum_{k^{\prime}=1}^{m_{j}} \frac{1}{p_{i, k^{\prime}, \mu(i, r)}}\right)
\end{aligned}
$$

Therefore, $T^{i *}$ is calculated by:

$$
T_{i}^{*}=\sqrt{\frac{\sum_{r=1}^{m_{i}} S_{i, k^{\prime}, \mu(i, r)}+A}{\left(\frac{d_{i}^{2}}{2} \sum_{r=2}^{m_{i}} h_{i, \mu(i, r-1)}\left(\frac{1}{p_{i, k^{\prime}, \mu(i, r)}}+\frac{1}{p_{i, k^{\prime}, \mu(i, r-1)}}\right)+\frac{h_{i} d_{i}}{2}\left(3-\frac{1}{p_{i, k^{\prime}, m_{i}}}\right)-h_{i} d_{i}{ }^{2} \sum_{r=1}^{m_{i-1}} \frac{1}{p_{i, k^{\prime}, \mu(i, r)}}\right)}}
$$

By considering other constraints, the global minimum of the cycle time is computed by: 
$T_{i}^{*}=\min$

$$
\begin{aligned}
& {\left[\max \left\{\sqrt{\left.\frac{d_{i}^{2}}{2} \sum_{r=2}^{m_{i}} h_{i, \mu(i, r-1)}\left(\frac{1}{p_{i, k^{\prime}, \mu(i, r)}}+\frac{1}{p_{i, k^{\prime}, \mu(i, r-1)}}\right)+\frac{h_{i} d_{i}}{2}\left(3-\frac{1}{p_{i, k^{\prime}, m_{i}}}\right)-h_{i} d_{i}{ }^{2} \sum_{r=1}^{m_{i-1}} \frac{1}{p_{i, k^{\prime}, \mu(i, r)}}\right)}, \max _{i=1, \ldots, n}\left\{\frac{\sum_{r=1}^{m_{i}} S t_{i k^{\prime} \mu(i, r)}}{1-\sum_{r=1}^{m i} \frac{d_{i}}{P_{i, k^{\prime}, \mu(i, r)}}}\right\}\right\},\right.} \\
& \left\{\min _{i=1, \ldots, n}\left\{\frac{L_{i}}{1-\sum_{r=1}^{m_{i}} \frac{d_{i}}{p_{i, k^{\prime}, \mu(i, r)}}}\right\}, H\right\}
\end{aligned}
$$

Step 2: Let all $k_{i}$ values be equal to one and assign and sequence all $k_{i}$ by the first available machine (FAM) rule described in Section 3.2. Therefore, this model is an NLP model with a common cycle approach where this model results is an upper bound (TCub) for the basic problem of this paper.

Step 3: Compute an upper bound for multipliers $k_{i}(i=1, \ldots, n)$ by the following equation,

$$
k_{i}^{u b}=\left\lceil\frac{T_{i}^{*}}{\min _{i=1, \ldots, n}\left\{T_{i}^{*}\right\}}\right\rceil ; i=1, \ldots, n
$$

where $\lceil v\rceil$ returns the smallest and integer number of greater than $v$. Make all combination of the $k_{i}$ that are lower than $k_{i}^{u b}$ and greater than one for different products. Then as described in Section 4.2, compute the $T C_{l b}$ as a lower bound of the total cost for each combination of the multipliers. If $T C_{l b} \leq T C_{u b}$, then store the set of multipliers as a candidate vector, else delete this set from the main list. Finally, at the end of this stage, all candidate vectors of the multipliers are determined.

\subsection{Determining sequence production in basic periods}

To assign and sequence different products in different basic periods, a method is defined to find a feasible sequence. Finding a sequence with a simple necessary and sufficient condition is not easy to derive; therefore; the method proposed by Torabi \& Jenabi (2009a) is used. For any candidate vector of the multipliers, sort products in vector $O^{\prime}$ in non-decreasing order of $k_{i}$ values. If there are some products with the same $k_{i}$ values, then sort these products in non-increasing order of $\rho_{i}$ values, where the $\rho_{i}$ calculation is as follows.

$\rho_{i}=\sum_{r=1}^{m_{i}} \frac{k_{i} d_{i}}{P_{i, \mu(i, r)}} ; i=1, \ldots, n$

Assign each product $i$ in the vector $O^{\prime}$ to the different basic period such that:

$\min \left\{\operatorname{Max}_{k=t, t+1, \ldots, H}\left\{\operatorname{Max}_{r=1, \ldots, m_{i}}\left(\sum_{u \in \sigma_{k}} \frac{k_{u} d_{u}}{p_{u, \mu(u, r)}}+\frac{k_{i} d_{i}}{p_{i, \mu(i, r)}}\right)\right\}\right\}$

Finally, for each candidate vector of multipliers, a sequence of products $\left(\sigma_{k}\right)$ is determined in each basic period, such that if $i$ and $u$ are in the sequence vector in basic period $k$-th, and $i$ is ordered before $u$ in $O^{\prime}$, then $i$ is also ordered before $u$ in $\sigma_{k}$.

\subsection{Assigning and sequencing products of each basic period on machines}

In this section, a procedure for sequencing and scheduling products in different basic periods is used namely the first available machine (FAM) rule. In other words, products are sequenced and scheduled based on their basic periods on different machines and according to in their own routes. The FAM rule 
is presented by Torabi et al. (2006), in which this method is used with some developments in this paper. In this procedure, for any given permutation vector $O^{\prime}$; because of the unrelated parallel machines in different stages, products are assigned to machines of the first stages on their routes (if $m_{1}>$ 1), randomly. Then, the products are assigned to the machines in each subsequent stage, when each product is finished its operation at the previous stages earlier, and finally the products are assigned to the machines at the current stage according to the FAM rule. If there is more than one machine available for assignment, the algorithm selects the machine at this stage, randomly.

\subsection{Fitness function}

Each candidate vector of $k_{i}$ values is sequenced in basic periods. Therefore, after sequencing each candidate vector, values of $X_{i l k^{\prime} k \mu(i, r)}$ in problem $P_{f l}$ and $P_{f u}$ are determined and it is one or zero in different positions by their sequence. Thus, two models $P_{f l l}$ and $P_{f u l}$ (NLP models) are resulted from the MINLP models of $P_{f l}$ and $P_{f u}$, respectively. Therefore, in this section, an algorithm is defined to solve these two models. The problem $P_{f u l}$ is as follows.

\section{Problem $P_{f u 1}$}

$\min P_{f u l}=\frac{\sum_{i=1}^{n} \Sigma_{r=1}^{m_{i}} \Sigma_{k^{\prime}=1}^{m_{j}} S_{i k^{\prime} \mu(i, r)}}{k_{i} \cdot F_{\alpha}^{U}}+\frac{A}{F_{\alpha}^{U}}+\sum_{i=1}^{n} \frac{d_{i}{ }^{2}}{2}\left(k_{i} F_{\alpha}^{U}\right) \cdot \sum_{r=2}^{m_{i}} h_{i, \mu(i, r-1)}\left(\sum_{k^{\prime}=1}^{m_{j}} \sum_{l=1}^{n_{j}} \frac{1}{p_{i, k^{\prime}, \mu(i, r)}}-\right.$

$\left.\sum_{k^{\prime}=1}^{m_{j-1}} \sum_{l=1}^{n_{j}} \frac{1}{p_{i, k^{\prime}, \mu(i, r-1)}}\right)+\sum_{i=1}^{n} \sum_{r=2}^{m_{i}} h_{i, \mu(i, r-1)} \cdot d_{i}\left(b_{i, \mu(i, r)}-b_{i, \mu(i, r-1)}\right)+F_{\alpha}^{U} \cdot \sum_{i=1}^{n} \frac{h_{i} d_{i} k_{i}}{2}(3-$ $\left.\frac{1}{p_{i, k^{\prime}, m_{i}}}\right)-\sum_{i=1}^{n} h_{i} d_{i} b_{i, \mu\left(i, m_{i}\right)}$

subject to

$$
\begin{aligned}
& b_{i, \mu(i, r-1)}+\frac{d_{i} \cdot k_{i} \cdot F_{\alpha}^{U}}{P_{i, k^{\prime}, \mu(i, r-1)}} \leq b_{i, \mu(i, r)} ; i=1, \ldots, n, r=2, \ldots, m_{i} ; k=1, \ldots, m_{j} \\
& b_{i, \mu(i, r)}+\frac{d_{i \cdot k_{i} \cdot F_{\alpha}^{U}}}{P_{i, k^{\prime}, \mu(i, r)}}+S t_{u, k^{\prime}, \mu(i, r)} \leq b_{u, \mu(i, r)} ; i \neq \mathrm{u}, i, \mathrm{u} \in \mathrm{p}(r) ; \mathrm{L}<n_{j} \\
& b_{i, \mu(i, r)} \geq S t_{i, k^{\prime}, \mu(i, r)} ; r=1, \ldots, m_{i} ; i \in \mathrm{n} ; k=1, \ldots, H ; H=\operatorname{LCM}\left(k_{1}, k_{2}, \ldots, k_{n}\right) \\
& b_{i, \mu\left(i, m_{i}\right)}+\frac{d_{i} k_{i} F_{\alpha}^{U}}{p_{i, k \prime, \mu\left(i, m_{i}\right)}} \leq F_{\alpha}^{L} ; i=1, \ldots, n
\end{aligned}
$$

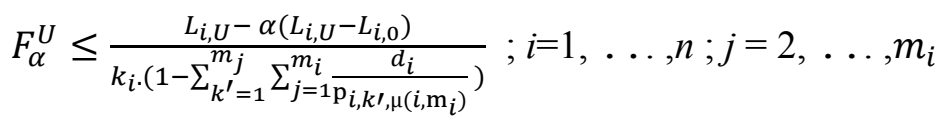

$$
\begin{aligned}
& H . F_{\alpha}^{U} \cdot R=P H ; H=\operatorname{LCM}\left(k_{1}, k_{2}, \ldots, k_{n}\right) \\
& R \geq 1 ; R=\text { integer } ; F_{\alpha}^{U} \geq 0 ; b_{i, j} \geq 0 \forall i, j ; 0 \leq \alpha \leq 1
\end{aligned}
$$

The iterative procedure is defined as follows:

Step 1: $\quad$ Let $R=1$ and calculate $F_{\alpha}^{U}$ value as $F_{\alpha}^{U}=P H / H . R$.

Step 2:If the resulted value of $F_{\alpha}^{U}$ is lower than shelf life constraints by considering $\alpha$ equal to zero $\left(F_{\alpha}^{U} \leq \frac{L_{i, U}}{k_{i} \cdot\left(1-\sum_{k^{\prime}=1}^{m_{j}} \sum_{j=1}^{m_{i}} \frac{d_{i}}{\mathrm{p}_{i, k^{\prime}, \mu\left(i, \mathrm{~m}_{i}\right)}}\right)} ; i=1, \ldots, n\right)$ go to step 3 , else increase $R$ by one $(R=R+1)$ and repeat step 2. 
Step 3: By determined $R$ in step 2, solve the linear problem, and set $Z$ to the objective value results, and then go to Step 4.

Step 4:Increase $R$ by 1 and solve $P_{f u l}$. If this model has no feasible solution, stop; else, if the objective function for current value of $R\left(Z_{R}\right)$ is less than the objective value for previous $R(Z)$, then set $Z=Z_{R}$ and $F_{\alpha}^{U^{*}}=P H / R . H$, and iterate Step 4.

The $P_{f l l}$ model is the same as the $P_{f u l}$, and the procedure to solve it is mostly similar to the procedure for the $P_{f u l}$ problem. The only difference is that in Step 2 in iterative procedure, $\alpha$ is equal to one and all $F_{\alpha}^{U}$ parameters are changed to $F_{\alpha}^{l}$.

\subsection{Defuzzify the solutions}

In this section, to convert the fuzzy solutions and objective functions of the two models of the $P_{f u}$ and $P_{f l}$, a maximum method is illustrated. This method is simple and change fuzzy values to crisp values easily. By this method, the final objective function is computed by:

$P_{f z}=\max \left\{P_{f u 1}, P_{f l 1}\right\}$

\subsection{Feasibility test and repair procedure}

This heuristic method solves all candidate vectors and at last selects the best one as the best solution of the problem. However, it is possible that some of the candidate vectors yield infeasible solutions. Thus, in this stage, an algorithm is used to repair infeasible candidate vectors to feasible. To control the feasibility test for each candidate vector, a simple test is defined. For the feasibility test, first, the process completion time $\left(\sum_{r=1}^{m_{i}} k_{i} d_{i} / p_{i, \mu(i, r)}\right)$ of each product for all $H$ basic periods is calculated. If this value is less than or equal to one, then this solution is feasible; otherwise if this value is greater than one even at least in one basic period, it is infeasible and needs the repair procedure. Greater than one means that the assignment for production of the product is more than the capacity. The repair procedure is shown as follows.

Step 1: Choose the products with the biggest process completion time.,

Step 2:If $k_{i} \neq 1$, set $k_{i}=k_{i}-1$, and obtain sequencing, scheduling and lot sizing the new problem. Else, if $k_{i}=1$, then select the next product with the biggest value of $\sum_{r=1}^{m_{i}} k_{i} d_{i} / p_{i, \mu(i, r)}$ for each product and go to Step 2,

Step 3: If this solution is feasible stop; otherwise, go to Step 1.

\section{Computational experiments and discussion}

To evaluate the efficiency of our proposed heuristic method, a comparison between our solutions and lower bound is made. Therefore, several numerical experiments with different values are conducted and implemented on a personal computer Intel core i3-2410 MB CPU. The proposed heuristic method is coded in MATLAB 9.

\subsection{Parameter setting}

To solve this problem by the proposed heuristic method, parameters are set as follows. The tuned parameters of this method after initial are adjusted and the planning horizon is at least equal to 52 weeks. Moreover, the parameters of the test problems are obtained from the following uniform distribution:

$$
d_{i} \sim U(100,1000), p_{i, \mu(i, r)} \sim U(5000,15000), S t_{i, \mu(i, r)} \sim U(0.01,0.025), h_{i, \mu(i, 1)} \sim U(1,10) A_{i} \sim U(10000,20000)
$$


Products have an added-up value after each operation in different stages on their routes; therefore, values of the $h_{i, \mu(i, r)}$ are non-decreasing order after each production stage. So, after generating $h_{i, \mu(i, 1)}$ for each product, $h_{i, \mu(i, r)}$ can be determined as $h_{i, \mu(i, r)}=h_{i, \mu(i, r-1)}+U(1,4)$.

To determine the setup time values, we assume that there is a correlation between setup times and setup costs. Thus, after generating setup times, the setup costs are defined by using $S_{i, j}=15000 \times S t_{i, j}+1000 \times U(0,1)$. In addition, $L_{i}$ values should be large enough to be feasible. Thus, it is assumed that this value should be greater than $F_{\min } . L_{i}, L_{i L}$ and $L_{i U}$ values are generated by:

$$
L_{i} \sim F_{\text {min }}+\text { rand } \times P H, \quad L_{i L} \sim F_{\text {min }}+\text { rand } \times L_{i}, L_{i U} \sim L_{i}+\text { rand } \times L_{i}
$$

The $F_{\min }$ means $F$ should be large enough to produce at least one product in all basic periods when all multipliers are equal. Thus, $F_{\min }$ is as follows:

$$
\operatorname{Max}_{i=1, \ldots, n}\left\{\sum_{r=1}^{m_{i}} S t_{i k^{\prime} \mu(i, r)}+\sum_{r=1}^{m i} \frac{F d_{i}}{P_{i, k^{\prime}, \mu(i, r)}}\right\} \leq F \rightarrow F_{m i n} \geq \operatorname{Max}_{i=1, \ldots, n}\left\{\frac{\sum_{r=1}^{m_{i}} S t_{i k^{\prime} \mu(i, r)}}{1-\sum_{r=1}^{m i} \frac{d_{i}}{P_{i, k^{\prime}, \mu(i, r)}}}\right\}
$$

\subsection{Performance evaluation}

To evaluate the efficiency of the proposed heuristic method, eight different problems are considered. For each problem size, 20 problems are generated randomly for evaluating the heuristic performance. The small-sized problems have 4 and 5 products with 2 and 3 production stages, and problem instances with 5 and 10 products with 5 and 10 stages are medium and large-sized problems. Because of a large computational time for the exact solution and dependency of the main model to the multipliers, the heuristic solution is compared with a lower bound. The $\Omega$ index as $\Omega=(T C-L B) / L B$ can be calculated as a lower bound. In this equation, $T C$ is an objective function that is received from the proposed heuristic method, and LB is the lower bound of the problem. To find $L B$, the following equation should be minimized.

$$
\begin{aligned}
& T C=\frac{\sum_{i=1}^{n} \sum_{r=1}^{m_{i}} s_{i k^{\prime} \mu(i, r)}}{k_{i} \cdot F}+\frac{A}{F}+\sum_{i=1}^{n} \frac{d_{i}{ }^{2}}{2}\left(k_{i} F\right) \cdot \sum_{r=2}^{m_{i}} h_{i, \mu(i, r-1)}\left(\frac{1}{p_{i, k^{\prime}, \mu(i, r)}}-\frac{1}{p_{i, k^{\prime}, \mu(i, r-1)}}\right)+ \\
& \sum_{i=1}^{n} \sum_{r=2}^{m_{i}} h_{i, \mu(i, r-1)} \cdot d_{i}\left(b_{i, \mu(i, r)}-b_{i, \mu(i, r-1)}\right)+F \cdot \sum_{i=1}^{n} \frac{h_{i} d_{i} k_{i}}{2}\left(3-\frac{1}{p_{i, k^{\prime}, m_{i}}}\right)- \\
& \sum_{i=1}^{n} h_{i} d_{i} b_{i, \mu\left(i, m_{i}\right)}
\end{aligned}
$$

By the constraints considered in the main model, $\left(b_{i, \mu(i, r)}-b_{i, \mu(i, r-1)}\right)$ and $b_{i, \mu\left(\mathrm{I}, m_{i}\right)}$ change to $d_{i} k_{i} F / P_{i k^{\prime} \mu(i, r-1)}$ and $F\left(1-d_{i} k_{i} / p_{i, k^{\prime}, \mu(i, m i)}\right)$ respectively, to minimize Eq. (46). Thus, $F_{l b}$ and $L B$ are computed as follows.

$$
\begin{gathered}
F_{l b}=\sqrt{\frac{A+\frac{\sum_{i=1}^{n} \sum_{r=1}^{m_{i}} S_{i k^{\prime} \mu(i, r)}}{k_{i}}}{\sum_{i=1}^{n} \frac{d_{i}^{2}}{2}\left(k_{i}\right) \cdot \sum_{r=2}^{m_{i}} h_{i, \mu(i, r-1)}\left(\frac{1}{p_{i, k^{\prime}, \mu(i, r)}}+\frac{1}{p_{i, k^{\prime}, \mu(i, r-1)}}\right)+\sum_{i=1}^{n} \frac{h_{i} d_{i} k_{i}}{2}\left(3-\frac{1}{p_{i, k^{\prime}, m_{i}}}\right)-\sum_{i=1}^{n} h_{i} d_{i}{ }^{2} \sum_{r=1}^{m_{i-1}} \frac{1}{p_{i, k^{\prime}, \mu(i, r)}}}} \\
L B=\frac{\sum_{i=1}^{n} \sum_{r=1}^{m_{i}} S_{i k^{\prime} \mu(i, r)}}{K_{i} F_{L B}}+\frac{A}{F_{L B}}+\sum_{i=1}^{n} \frac{d_{i}^{2}}{2}\left(k_{i} F_{L B}\right) \cdot \sum_{r=2}^{m_{i}} h_{i, \mu(i, r-1)}\left(\frac{1}{p_{i, k^{\prime}, \mu(i, r)}}+\frac{1}{p_{i, k^{\prime}, \mu(i, r-1)}}\right)+F_{L B} \cdot \sum_{i=1}^{n} \frac{h_{i} d_{i} k_{i}}{2}(3- \\
\left.\frac{1}{p_{i, k^{\prime}, m_{i}}}\right)-\sum_{i=1}^{n} F_{L B} \cdot h_{i} d_{i}{ }^{2} \sum_{r=1}^{m_{i-1}} \frac{1}{p_{i, k^{\prime}, \mu(i, r)}}
\end{gathered}
$$

Tables 1 present the computational results of the problem. In each cell of this table, four entries are illustrated, which are the minimum, the maximum, the standard deviation and the mean of $\Omega$ and 
percentage performance ratio. In this table, the solutions of the proposed heuristic method are compared with the common cycle time solutions. To solve the problem with the common cycle time approach, it is just needed to assume all $k_{i}$ values be equal to one and solve the problem with the proposed heuristic method. The associated results have been compared with the solutions of the problem without considering the shelf life constraint. In addition, the average performance of the proposed heuristic solutions is about $8.55 \%$ over the common cycle time solutions. The $\Omega$ index is illustrated in the Table 1. As it is clear, the difference between the lower bound and problem solutions is larger in medium and large-sized problems. This increase is maybe for increasing the solution space sensible. Furthermore, when the shelf life constraint is eliminated from the basic model, the proposed heuristic method gives better optimal solutions over the common cycle approach.

Table 1

Computational results

\begin{tabular}{|c|c|c|c|c|c|}
\hline \multirow[b]{2}{*}{$\begin{array}{l}\text { Problem size } \\
\qquad(n \times m)\end{array}$} & & \multirow[b]{2}{*}{$\Omega(\%)$} & \multirow[b]{2}{*}{ A } & \multicolumn{2}{|c|}{ Without considering the shelf life constraint } \\
\hline & & & & $\Omega(\%)$ & $\mathrm{B}$ \\
\hline \multirow[t]{4}{*}{$4 \times 2$} & Minimum & 0.03 & 3.24 & 0.03 & 6.88 \\
\hline & Maximum & 10.35 & 24.35 & 8.43 & 15.79 \\
\hline & S.D. & 3.05 & 7.23 & 2.55 & 4.88 \\
\hline & Mean & 3.91 & 11.56 & 3.33 & 6.35 \\
\hline \multirow[t]{4}{*}{$4 \times 3$} & Minimum & 0.42 & 1.29 & 1.29 & 3.20 \\
\hline & Maximum & 13.49 & 27.45 & 11.25 & 27.86 \\
\hline & S.D. & 3.58 & 8.23 & 2.98 & 8.79 \\
\hline & Mean & 3.86 & 11.13 & 3.17 & 13.86 \\
\hline \multirow[t]{4}{*}{$5 \times 2$} & Minimum & 0.64 & 2.34 & 0.62 & 2.85 \\
\hline & Maximum & 15.70 & 19.35 & 10.60 & 21.99 \\
\hline & S.D. & 4.48 & 7.74 & 4.42 & 7.79 \\
\hline & Mean & 4.47 & 11.01 & 4.12 & 13.88 \\
\hline \multirow[t]{4}{*}{$5 \times 3$} & Minimum & 0.85 & 1.19 & 0.78 & 2.88 \\
\hline & Maximum & 27.92 & 16.94 & 20.51 & 19.76 \\
\hline & S.D. & 5.84 & 6.34 & 4.85 & 8.99 \\
\hline & Mean & 5.52 & 8.90 & 4.76 & 10.75 \\
\hline \multirow[t]{4}{*}{$5 \times 5$} & Minimum & 1.01 & 2.32 & 0.94 & 3.88 \\
\hline & Maximum & 25.08 & 18.23 & 15.84 & 19.79 \\
\hline & S.D. & 6.16 & 5.34 & 4.68 & 6.87 \\
\hline & Mean & 6.12 & 9.53 & 5.16 & 11.87 \\
\hline \multirow[t]{4}{*}{$5 \times 10$} & Minimum & 0.72 & 1.34 & 0.69 & 2.86 \\
\hline & Maximum & 46.14 & 14.35 & 33.58 & 16.99 \\
\hline & S.D. & 12.62 & 4.23 & 9.74 & 5.88 \\
\hline & Mean & 12.69 & 6.23 & 11.29 & 7.88 \\
\hline \multirow[t]{4}{*}{$10 \times 5$} & Minimum & 1.05 & 2.45 & 1.05 & 5.79 \\
\hline & Maximum & 35.83 & 13.35 & 25.86 & 15.88 \\
\hline & S.D. & 10.59 & 3.34 & 8.45 & 4.12 \\
\hline & Mean & 14.37 & 5.73 & 12.66 & 6.98 \\
\hline \multirow[t]{4}{*}{$10 \times 10$} & Minimum & 8.75 & 1.01 & 8.17 & 2.88 \\
\hline & Maximum & 30.23 & 12.12 & 25.37 & 15.88 \\
\hline & S.D. & 9.35 & 2.73 & 10.36 & 4.87 \\
\hline & Mean & 18.55 & 4.32 & 15.84 & 6.99 \\
\hline
\end{tabular}

A: Percentage performance ratio of the proposed heuristic solution comparing to the common cycle solution.

B: Percentage performance ratio of the proposed heuristic solution comparing to the common cycle solution without considering the shelf life constraint 
Table 2 presents the computational time results. To compare these results, we also solve the problem with crisp shelf life values and compare the results with the Lingo solutions of the common cycle time approach. For another comparison, the results of the problem are compared with the optimal enumeration method (OEM) presented by Karimi et al. (2005) in a flexible job shop environment under the common cycle time approach. This method gives the optimal solutions for small and medium-sized problems. However, this method has non-polynomial complexity at each step, and this method do not solve the problem in a reasonable CPU time. We have solved our problem by the OEM without considering the shelf life constraint and under common cycle time approach and the results are shown in Table 2.

About the computational time, when the problem sizes are small, the proposed heuristic is relatively effective. However, when the problem size is larger, the computational time suddenly increases. Let the upper bound for $k_{i}$ values in small, medium and large-sized problems be all equal to 3 . When the heuristic method solves the problem with four or five products, it has to compute at least 81 (i.e., equal to $3^{4}$ ) and maximum 243 (i.e., equal to $3^{5}$ ) potential solutions and control them for the heuristic solutions. However, in large-sized problems with ten products, the proposed heuristic method has at least 59049 potential solutions (i.e., equal to $3^{10}$ ).

Thus, it can be concluded that this heuristic method for small-sized problems works well; however, it needs a significant amount of computational time for large-sized problems. In comparison with OEM and Lingo solutions, the proposed heuristic maintains better computational time. As it is clear in Table 2 , it maybe OEM has better computational time in some cases like problem sizes $5 \times 2$ and $5 \times 3$; however, it has to be mentioned the proposed model under the basic period approach gives better solutions totally over the common cycle approach, as it is illustrated in Table 1.

Table 2

Computational time results (sec.)

\begin{tabular}{|c|c|c|c|c|c|}
\hline Problem size $(n \times m)$ & & A & B & Lingo & OEM \\
\hline \multirow[t]{2}{*}{$4 \times 2$} & Mean & 43.19 & 18.41 & 2927.24 & 34.35 \\
\hline & S.D. & 12.46 & 8.32 & 339.79 & 9.62 \\
\hline \multirow[t]{2}{*}{$4 \times 3$} & Mean & 66.26 & 37.77 & 4303.68 & 46.88 \\
\hline & S.D. & 14.32 & 10.85 & 624.45 & 10.31 \\
\hline \multirow[t]{2}{*}{$5 \times 2$} & Mean & 130.62 & 70.32 & 5209.91 & 54.23 \\
\hline & S.D. & 28.35 & 16.45 & 1356.92 & 11.93 \\
\hline \multirow[t]{2}{*}{$5 \times 3$} & Mean & 150.22 & 84.12 & 6438.09 & 66.23 \\
\hline & S.D. & 33.57 & 14.28 & 2898.09 & 19.87 \\
\hline \multirow[t]{2}{*}{$5 \times 5$} & Mean & 174.69 & 64.63 & _ & 104.55 \\
\hline & S.D. & 45.32 & 22.76 & & 35.55 \\
\hline \multirow[t]{2}{*}{$5 \times 10$} & Mean & 251.27 & 143.23 & - & 165.37 \\
\hline & S.D. & 66.95 & 36.57 & & 38.03 \\
\hline \multirow[t]{2}{*}{$10 \times 5$} & Mean & 6110.50 & 2627.52 & - & 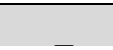 \\
\hline & S.D. & 1870.76 & 343.88 & - & \\
\hline \multirow[t]{2}{*}{$10 \times 10$} & Mean & 8041.29 & 4503.12 & - & 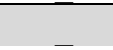 \\
\hline & S.D. & 1343.36 & 762.46 & & \\
\hline \multirow[t]{2}{*}{ All test problems } & Mean & 1871.01 & 943.64 & - & - \\
\hline & S.D. & 653.33 & 323.45 & & \\
\hline
\end{tabular}

\section{Conclusions}

In this paper, the basic period approach has been considered to solve the economic lot and delivery scheduling problem in a flexible job shop with unrelated parallel machines considering a fuzzy shelf 
life. A basic mixed-integer nonlinear programming (MINLP) model has been presented and then developed for fuzzy models. Because of the dependency of the model to the $k_{i}$ values, solving the fuzzy models has been impossible directly. Therefore, a heuristic method has been proposed to search all feasible and potential values of $k_{i}$. The first available machine (FAM) rule has been developed for sequencing the flexible job shop with unrelated parallel machines. By the obtained results, it has been shown that the proposed heuristic method has been suitable for small and large-scale problems. When the product number of the problem increases, the efficiency of the proposed method decreases while the CPU computation time increases. In addition, it has been found that the proposed method has found better solutions over lower bounds and the common cycle approach solutions.

\section{Acknowledgment}

The authors would like to thank the anonymous referees for constructive comments on earlier version of this work.

\section{References}

Bomberger, E. (1966). A dynamic programming approach to a lot size scheduling problem. Management Science, 12(11), 778-784.

Chang, P.T., Yao, M.J., Huang, S.F, \& Chen, C.T. (2006). A genetic algorithm for solving a fuzzy economic lot-size scheduling problem. International Journal of Production Economics, 102, 265288.

El-Najdawi, M. K., \& Kleindorfer, P.R. (1993). Common cycle lot size scheduling for multi-product, multi-stage production. Management science, 39(7), 872.

Fatemi Ghomi, S.M.T., \& Torabi, S.A. (2002). Extension of common cycle lot size scheduling for multi-product, multi-stage arborscent flow-shop environment. Iranian Journal of Science and Technology, 26(B1): 55-68.

Haessler, R. (1979). An improved extended basic period procedure for solving the economic lot scheduling problem. AIIE Transactions, 11(4): 336-340.

Hahm, J., \& Arai Yanob, C. (1992). The economic lot and delivery scheduling problem: the single item case. International Journal of Production Economics, 28(2): 235.

Hahm, J., \& Yano, C.A. (1995). The economic lot and delivery scheduling problems: models for nested schedules. IEE Transactions, 27(2): 126-139.

Hanssmann, F. (1962). Operations research in production and inventory control, John Wiley \& Sons.

Jenabi, M., Fatemi Ghomi,S., Torabi, S., \& Karimi,B. (2007). Two hybrid meta-heuristics for the finite horizon ELSP in flexible flow lines with unrelated parallel machines. Applied Mathematics and Computation, 186(1), 230-245.

Lee, H. M., \& Yao, J.S. (1998). Economic production quantity for fuzzy demand quantity and fuzzy production quantity. European Journal of Operational Research,109, 203-211.

Lin, D.C., \& Yao, J.S. (2000). Fuzzy economic production for production inventory. Fuzzy Sets and Systems, 111, 465-495.

Liu, J., Wu, L. , \& Zhou, Z. (2008). A time-varying lot size method for the economic lot scheduling problem with shelf life considerations. European Journal of Industrial Engineering, 2(3), 337-355.

Lütke entrupa, M., Günthera, H.O., Van Beekb, P.,Grunowa, M., \& Seilera,T. (2005). Mixed-Integer Linear Programming approaches to shelf-life-integrated planning and scheduling in yoghurt production. International Journal of Production Research, 43(23), 5071-5100.

Mokhlesian, M., Fatemi Ghomi, S.M.T., Jolai, F. (2010). Economic lot scheduling problem with consideration of money time value. International Journal of Industrial Engineering Computations, 1(2), 121-138.

Ouenniche, J., \& Bertrand, J.W.M. (2001). The finite horizon economic lot sizing problem in job shops:the multiple cycle approach. International Journal of Production Economics, 74(1-3), 49-61. 
Ouenniche, J., Boctor, F. (1998). Sequencing, lot sizing and scheduling of several components in job shops: the common cycle approach. International Journal of Production Research, 36(4), 11251140.

Ouenniche, J., Boctor, F., \& Martel, A. (1999). The impact of sequencing decisions on multi-item lot sizing and scheduling in flow shops. International Journal of Production Research, 37(10), 22532270.

Ouenniche, J., \& Boctor, F. (2001a). The G-group heuristic to solve the multi-product, sequencing, lot sizing and scheduling problem in flow shops. International Journal of Produsction Research, 39(1), 81-98.

Ouenniche, J., \& Boctor, F. (2001b). The multi-product, economic lot sizing problem in flow shops: the powers-of-two heuristic. Computers \& Operations Research, 28(12), 1165-1182.

Ouenniche, J., \& Boctor, F. (2001c). The two-group heuristic to solve the multi-product, economic lot sizing and scheduling problem in tow shops. European Journal of Operational Research, 129(3), 539-554.

Pappis, C.P., \& Karacapilidis, N.I. (1995). Lot size scheduling using fuzzy numbers. International Transactions in Operational Research, 2: 205-212.

Rao, V.D.P., Subbaiah, K.V., Raju, V.R. (2009). Fuzzy Genetic Approach to Economic Lot Size Scheduling Problem. Jordan Journal of Mechanical and Industrial Engineering, 3, 9-16.

Roger, J. (1958). A Computational approach to the lot scheduling problem. Management science,4(3), 264-291.

Roy, T. K., \& Maiti, M (1997). A fuzzy EOQ model with demand-dependent unit cost under limited storage capacity. European Journal of Operational Research,99, 425-432.

Sarker, B., \& Babu, P. (1993). Effect of Production on shelf life. International Journal of Production research, 31(8), 1865-1872.

Sarker, B. R. (1994). Discussion a reply to A note on Effect of production cost on shelf life. International Journal of Production Research, 32(9), 2247.

Shaha, N.H., Pareek, S. and Sangal, I. (2012). EOQ in fuzzy environment and trade credit. International Journal of Industrial Engineering Computations, 3(2), 133-144.

Silver, E.A. (1989). Shelf life considerations in a family production context. International Journal of Production Research, 27(12), 2021-2026.

Sliver, E.A. (1995). Dealing with a shelf life constraint in cyclic shceduling by adjusting both cycle timeand production rate. International Journal of Production Research, 33(3), 623-629.

Soman, C., Van Donk,D., \& Gaalman,G. (2004). A basic period approach to the economic lot scheduling problem with shelf life considerations. International Journal of Journal Research, 42(8), 1677-1689.

Tavakkoli-Moghaddam, R., Azarkish, M., \& Sadeghnejad, A. (2011). Solving a multi-objective job shop scheduling problem with sequence-dependent setup times by a Pareto archive PSO combined with genetic operators and VNS. International Journal of Advanced Manufacturing Technology, 53, 733-750.

Torabi, S., \& Jenabi, M. (2009a). A meta-heuristic approach for the ELDSP in flexible flow lines: the power-of-two policy. Journal of Industrial Engineering, University of Tehran, 43(1), 1-13.

Torabi, S., \& Jenabi, M. (2009b). Multiple cycle economic lot and delivery-scheduling problem in a two-echelon supply chain. International Jounalof Advanced Manufacturing Technology, 43(7-8), 785-798.

Torabi, S.A., Karimi, B., \& Fatemi Ghomi, S.M.T. (2005). The common cycle economic lot scheduling in flexible job shops: The finite horizon case. International Journal of Production Economics, 97(1), 52-65.

Torabi, S.A., Karimi, B., \& Fatemi Ghomi, S.M.T. (2006). A hybrid genetic algorithm for the finite horizon economic lot and delivery scheduling in supply chains. European Journal of Operational Research, 173(1), 173-189.

Viswanathan, S. (1995). A note on effect of production cost on shelf life. International Journal of Production Research, 33(12), 3485-3486. 
Viswanathan, S., \& Goyal, S.K. (1997). Optimal cycle time and production rate in a family production context with shelf life considerations. International Journal of production Research, 35(6), 1703 1711.

Vujosevic, M., Petrovic, D., \& Petrovic, R. (1996). EOQ formula when inventory cost is fuzzy.International Journal of production Economics, 45, 499-504.

Yao, M.J., Chang, P.J., \& Huang, S.F. (2005). On the Economic Lot Scheduling Problem with Fuzzy Demands. International Journal of Operations Research, 2, 58-71.

Ziaeifar, A., Tavakkoli-Moghaddam, R., \& Pichka, K. (2011). Solving a new mathematical model for a hybrid flow shop scheduling problem with a processor assignment by a genetic algorithm. International Journalof Advanced Manufacturing Technology, DOI: 10.1007/s00170-011-3701-z. 